\title{
PSYCHO-ONCOLOGY: RATIONALE AND ITS PAKISTANI PERSPECTIVE
}

Psycho-oncology describes the psychological, social, behavioural and ethical aspects of cancer. It deals with the responses of patients and that of their families to cancer at all stages of the disease. Psycho-oncology also emphasizes psychological, behavioural and social factors that may influence the disease process.

\section{Brief History of Psycho-oncology}

Psycho-oncology formally came into existence around the mid-1970s when the stigma of having cancer was reduced and patient's feelings about their illness could be discussed for the $1^{\text {st }}$ time. The stigma of negative attitudes associated with mental illness and psychological problems has resulted in the delayed development of interest in psychological aspects of cancer. Psycho-oncology became a specialty of oncology with its own body of knowledge helping cancer care, in the last quarter of the past century. From the 1970s to 2000 following developments in the field of psychooncology were seen:

- First national conference on psychosocial research in the US in 1975.

- British psychosocial oncology society started in 1982.

- American psycho-oncology society started in 1986.

- Handbook of psycho-oncology published in 1989.

- Psycho-oncology journal published in 1992.

- Standards of care and clinical practice guidelines for psychosocial distress came out in 1998.

- First department of psychiatry and behavioural sciences was established in a cancer centre in 1996.

- The American College of Surgeon's Commission on Cancer established new accreditation standards for patient-centred care, including a mandate requiring that all patients be screened for distress with an appropriate intervention when patients are identified as distressed in 2007.

- The International Psycho-Oncology Society stated that distress should be included in the list of vital signs used to assess patients in 2009.

Correspondence: Dr. Sheheryar Jovindah,

Department of Internal Medicine, Consultant Child, Adolescent and Adult Psychiatrist, Shaukat Khanum Memorial Cancer Hospital and Research Centre, Lahore, Pakistan. Email: sheharyarj@skm.org.pk
At present, psycho-oncology departments or services exist as part of a comprehensive cancer centre in the developed countries. This consists of multidisciplinary team which includes psychiatrists, clinical psychologists, nutritionists, cancer specialist nurses and other allied health-care professionals.

\section{Application of Psycho-oncology in Clinical Practice}

In 1997, the National Comprehensive Cancer Network developed its first consensus-based guidelines to help healthcare professionals identify and treat patients with cancer who were in psychosocial distress. The term 'distress' was used to describe the multitude of complex symptomatology that deals with psycho-oncology and to avoid the use of stigmatizing terminology. Examples of distress include but not limited to financial hardship, family conflict, substance abuse issues or any issue that causes pain and anguish. The guidelines provide evidence-based information and clinical assessment tools to screen, diagnose and manage distress in patients diagnosed with cancer.

Evidence from clinical studies suggests benefit from on-going distress management in patients with cancer. A recent study showed that patients undergoing cancer treatment that received distress screening and followup tended to have less emergency room visits and hospitalizations. ${ }^{[1]}$ In this study, there was an $18-19 \%$ decline in the use of emergency and hospital services when there was an overall adherence to the distress screening protocol at community oncology centres.

A meta-analysis by DiMatteo et al. showed that noncompliance to therapy was 3 times higher in patients with clinical depression compared to those without depression. ${ }^{[2]}$ Furthermore, the presence of distress in patients' leads to poor decision-making, adversely affecting their quality of life and survival. A randomized study showed that initial distress screening with referral to psychosocial resources as appropriate led to reduced levels of distress at 3 months compared to screening without referrals. ${ }^{[3]}$ There is evidence from randomized studies that show improvement in the overall survival resulting from effective psychosocial interventions. ${ }^{[4-6]}$ 
Thus, early detection and intervention for distress management leads to better compliance with the therapy, improved communication and patient satisfaction and fewer use of medical services in patients with cancer.

\section{Psycho-oncology in Pakistan}

Psychological problems in Pakistan are grossly underaddressed. ${ }^{[7]}$ No surprise that psycho-oncology in Pakistan is in its infancy. Very few hospitals and cancer centres have any form of a formal psycho-oncology service.

In Pakistan, the prevalence of psychiatric and psychological problems in cancer patients is significantly higher than the developed nations. Various factors are responsible for this situation, some of these include:

- Significant stigma attached to mental health problems on part of the patients, family and even the treating doctors.

- The poor identification of psychiatric and psychological problems by doctors due to lack of education and awareness. Some cultural norms of minimizing psychiatric problems also have a role to play.

- There is a long list of stresses in the lives of cancer patients which include financial, social and family problems which contribute to the higher rate of psychiatric problems. The family support system in the urban areas is disintegrating resulting in poor support system for the physically and psychologically feeble cancer patients.

In Pakistan, unfortunately, the stress levels in the life of an ordinary person are gradually increasing resulting in more psychological distress, especially if they are diagnosed with cancer. As a result, robust, comprehensive and efficient psycho-oncology services are needed in every cancer centre of this country both in the public and private sectors.

Shaukat Khanum Memorial Cancer Hospital and Research Centre (SKMCH and RC), the first of its kind tertiary care cancer hospital has been offering services to cancer patient through clinical physiologists since its early years in 1995. In 2011, SKMCH and RC introduced a more comprehensive service by inclusion of consultant psychiatrist in the faculty. The psychooncology teamworks in a multidisciplinary setting with the oncologists, nutritionists, physiotherapist, play therapist and specialist cancer nurses. The psycho-oncology services are rendered through outpatient clinics and in the inpatients. Along with pharmacotherapy, the psychooncology team provides counselling and therapy to cancer patients who are in distress. Common psychological problems encountered include adjustment disorders, anxiety, depression, grief, pain and non-adherence to treatment. Focused support is provided through breast cancer and stoma support groups. Special support is also provided to paediatric patients and their families.

The Aga Khan University Hospital (AKUH), Karachi, one of the major tertiary care hospitals in the country also offers a psycho-oncology service collaboration between the Department of Oncology and Psychiatry. AKUH conducted a workshop on psycho-oncology in 2016 to highlight the importance of the discipline.

Psycho-oncology services at SKMCH and RC and AKUH are a good starting point for the development of the subspeciality in Pakistan - there is light at the end of the tunnel.

Sheheryar Jovindah ${ }^{1}$, Hassan Shahryar Sheikh ${ }^{2}$ ${ }^{1}$ Department Internal Medicine, Shaukat Khanum Memorial Cancer Hospital and Research Centre, Lahore, Pakistan, ${ }^{2}$ Department of Medical Oncology, Shaukat Khanum Memorial Cancer Hospital and Research Centre, Lahore, Pakistan

Received: 20 September 2017/Accepted: 27 September 2017

\section{References}

1. Zebrack B, Kayser K, Bybee D, et al. A practice-based evaluation of distress screening protocol adherence and medical service utilization. J Natl Compr Canc Netw 2017;15:903-12.

2. DiMatteo MR, Lepper HS, Croghan TW. Depression is a risk factor for noncompliance with medical treatment: Meta-analysis of the effects of anxiety and depression on patient adherence. Arch Intern Med 2000;160:2101-7.

3. Carlson LE, Groff SL, Maciejewski O, et al. Screening for distress in lung and breast cancer outpatients: A randomized controlled trial. J Clin Oncol 2010;28:4884-91.

4. Spiegel D. Mind matters in cancer survival. Psychooncology 2012;21:588-93.

5. Xia Y, Tong G, Feng R, et al. Psychosocial and behavioural interventions and cancer patient survival again: Hints of an adjusted meta-analysis. Integr Cancer Ther 2014;13:301-9.

6. Mustafa M, Carson-Stevens A, Gillespie D, et al. Psychological interventions for women with metastatic breast cancer. Cochrane Database Syst Rev 2013;6:CD004253.

7. Amin A, Gadit M. Mental health in Pakistan: Where do we stand? J Pak Med Assoc 2006;56:198-9. 